\title{
Effect of Cognitive Training by Music Therapy
}

\author{
Kazue Sawami $^{1 *}$, Tetsuro Kitamura ${ }^{1}$, Chizuko Suishu ${ }^{2}$ \\ ${ }^{1}$ Nara Medical University, Kashihara, Japan \\ ${ }^{2}$ Shubun University, Kashihara, Japan \\ "Corresponding Author: Kazue Sawami, Nara Medical University, Kashihara, Japan, Tel: +81744223051; E-mail: \\ sawami@naramed-u.ac.jp
}

Received: 22 October 2018; Accepted: 01 November 2018; Published: 05 November 2018

\begin{abstract}
Purpose: Targeting elderly people who live in local areas, by intervening the mixture of music therapy and recognition training, we will clarify if there is a significant difference among before the intervention, and nonintervened term, and after the intervention.
\end{abstract}

Method: The object of studies turned out to become 162 people out of 200 people through the invite applications. We compared the music therapy as once a month for 90 minutes with the group that were intervened for 3 months and the group that were not intervened for 3 months. We conducted the measurement between Montreal Cognitive Assessment test (MoCA test), which is a screening scale of mild cognitive impairment (MCI) and the measurement of the stress level was conducted by doing a comparative test by measuring the amylase of sublingual glands and one-way analysis of variance. In addition, we validated the interphase of cognitive function and the stress level with the use of Pearson's product moment correlation coefficient.

Result: Regarding cognitive scores, both immediate replay tasks $(\mathrm{p}<0.05)$ and delay playback tasks $(\mathrm{p}<0.01)$ had a significant rise after the intervention. In the respect of evaluating the total points of the doubt about the presence of MCI, the score improved after the intervention $(\mathrm{p}<0.01)$. The amount of amylase included in the saliva that shows the stress level decreased after the intervention $(\mathrm{p}<0.05)$, and there was a correlation found between the stress level and the cognitive scores.

Conclusion: After conducting the recognition training that integrates music therapy, there was a significant improvement in the cognitive score and a decrease in the stress level. Thus, this method is beneficial in terms of 
elderly people to do training without being stressed and from now on we will have to continue to improve the method.

Keywords: Music therapy; Cognitive ability; Stress level; Elderly people

\section{Introduction}

Alzheimer's disease: $\mathrm{AD}$ occupies more than 60\% in dementia, and the prevalence rate is rapidly increasing [1], which leads to the estimation that the prevalence rate of Japanese elderly people who are over 65 years old in 2012 , which is $15 \%$ is going to increase by $20 \%$ by 2025 [2]. At the early stage of AD, the composure of Amyloid $\beta$, which is the main component of senile plaques, but for the remedy that contrasts with the Amyloid $\beta$, we cannot see improvement in cognitive ability currently [3-4]. Therefore, under the present circumstance, the most important thing is to decrease the number of $\mathrm{AD}$ risk factor, and prevent them.

As a method of cognitive training to maintain the cognitive ability is verified that dual-task (practicing two tasks simultaneously) [5-6] and n-back task (to delay recall the nth time task) [7] is efficient. About the dual-task, in order to achieve the two tasks at the same time, it is necessary for the function of the frontal lobe that has prefrontal cortex as the center [8], and this thought that this leads to the training of the frontal lobe. In fact, it is verified from the examination that used the brain wave or the near-infrared spectroscopy that the frontal lobe became activated [9-10]. As for the n-back subject, the fact that was introduced by Wayne Kirchner in 1958, which states that even though it is a test of temporary memory, not only as an assessment test [11], but also it became clear that there is an effect of improvement in memorization and began to be used as a method for cognitive training.

Facts such as the improvement of fluid intelligence [12], the increase in the density of dopamine [13], and according to the meta-analysis, the frontal lobe and the activation of the area of parietal cortex has been verified [14]. In addition, the relevance between music and memorization is known [15], that music encourages the recollection of memories [16-18], and moreover it promotes the maintenance of the memories [19-21]. Furthermore, music improves the encoding capability of people's memory, which is to recognize the lyrics [22]. From these research results, the invention of programs for memory improvement using music therapy is progressing [23-24]. The characteristics of music therapy is that not only listening to music, but also the use of songs, dance, or instruments, and the combination of rhythmical movements. Because of the rhythmical movements, the serotonin, which is the neurotransmitter in the brain, is known to become active. Serotonin influences greatly on people's mental, and is relevant to the stableness in both mentally and physically and the calmness in people's emotions [25-26]. It is verified that serotonin also has an impact on the memory and learning effect among hippocampus [27-28].

In addition, the music therapy has the characteristic of achieving the dual-task, such as singing and memorizing the choreography at the same time. Moreover, we included delay play back tasks in it. This aims to gain synergies of the promotion in memorization and the memory tasks that is caused by the music therapy. It is easy to memorize the 
lyrics that goes along the music or ones that are on the beat, and it is know that it is difficult to memorize it when there is no music or rhythm. Particularly, the method called ostinato, which is to repeat the rhythm and the phrase, especially makes it unforgettable and is often contained in the music that beats time with using hands or legs [2930]. The main purpose of this research is to verify the improvement of cognitive ability with the use of the combination of music therapy and delay play back tasks.

Next, in order to reduce the negative influences that impact mentally or the cognitive ability that stems from the intervention within a group or stress that comes from memorization tasks [31], it is considered that the promotion of relaxation that is caused by the power of music is efficient [32], and we thought that the reduction of the stress level to be the second important aim. The fact of music as the response to stress, the decrease in the stimulation of anxiety, anger, or the sympathetic nerve is verified [33]. In this research, we have been conducting the intervention test for three months and compared the results of the intervention term with the contrast term.

\section{Method}

\subsection{The method of the subjects}

We recruited the subjects through publicity papers of universities, or through the distributions of posters at selfgovernment associations, and for the ones who were willing to register, we made them send an E-mail, or FAX, or call us.

\subsection{Lectotype}

Nothing else but only the ones who are able to walk by themselves. The number of subjects: 162 out of 200 people enrolled. Music therapy takes place once a month for 90 minutes every time (Figure 1).

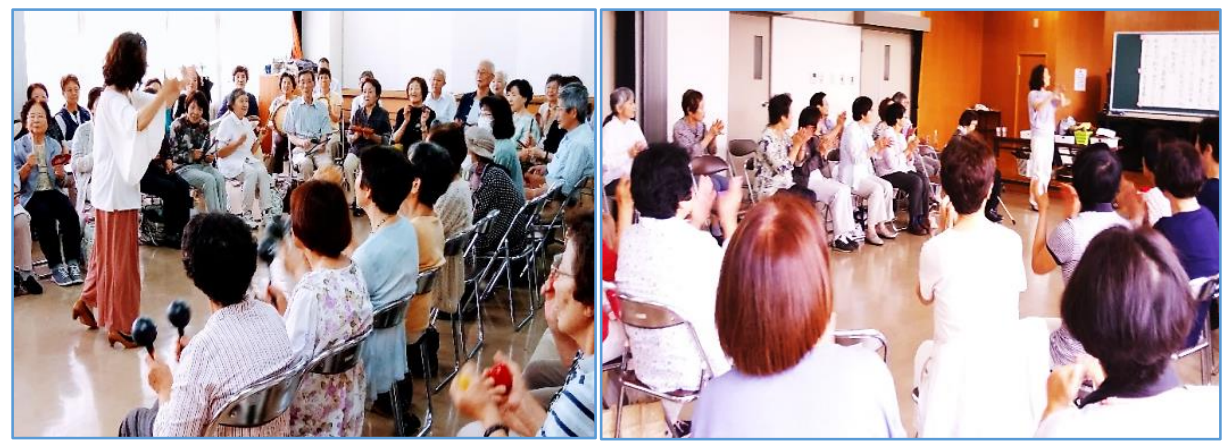

Figure 1: Cognitive training using music therapy.

Singing to the piano accompaniment and making sounds from each part. In addition to the musical phrases that you have memorized, you will have to memorize the ones after the performance, and memorize the choreography of the repeated pattern (delay recall tasks). Conduct different movements with left and right and also change the movements during the beat with their hands and the move of their steps (dual task). 


\subsection{The term}

March 2016-October.

2.4 The scale of measurement

2.4.1 The screening inspection of mild cognitive impairment: Montreal cognitive test (MoCA test) Figure 2.

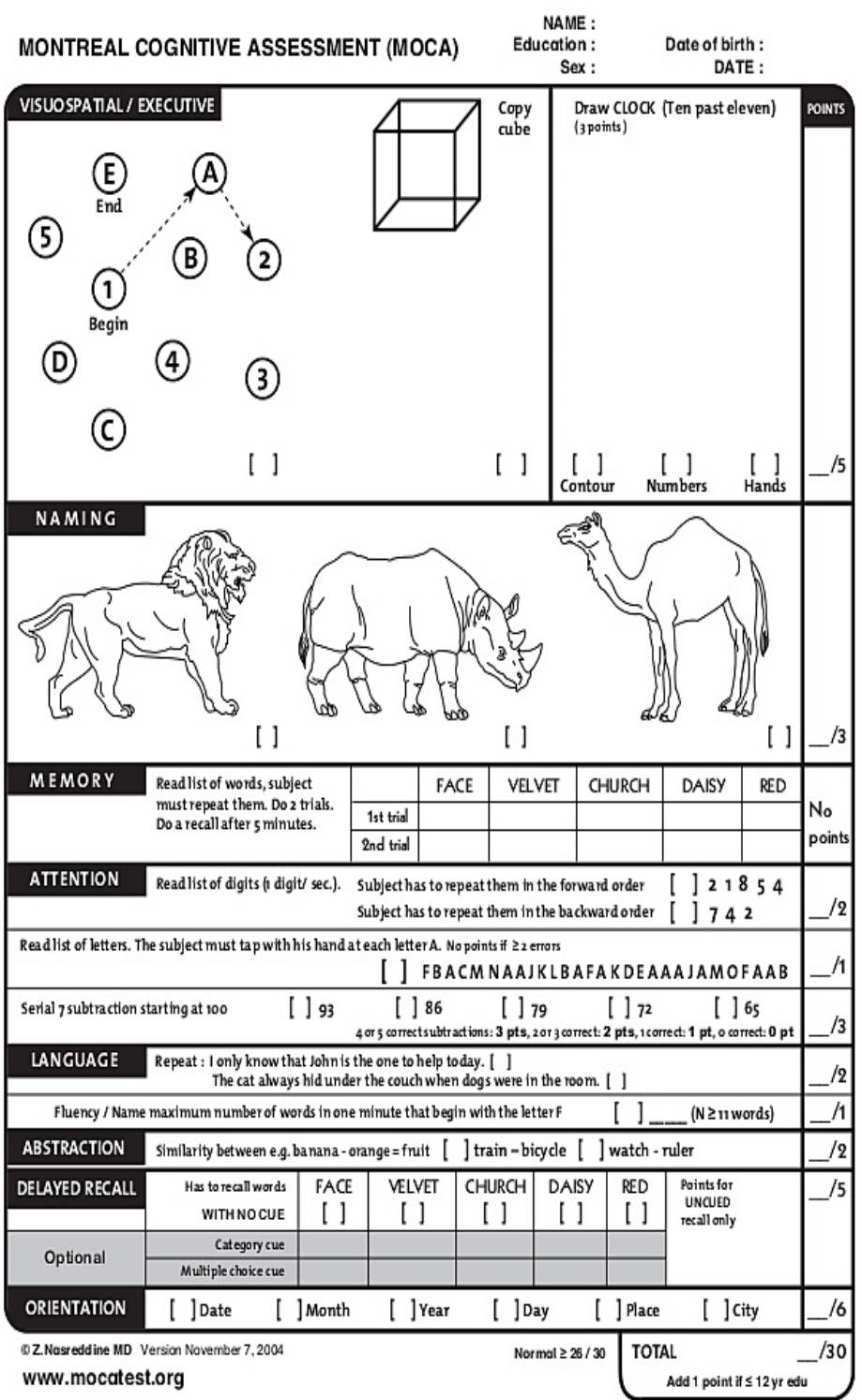

Figure 2: MoCA test. It is 30 points as a total it is the screen scale of mild cognitive impairment. As the total score is higher, the cognitive function is better, and the cut off value is 26 points. We have the permission of Dr. Ziad Nasreddine the inventor when using it. Authority: www.mocatest.org. 
2.4.2 The check of stress: The measurement of the amount of sublingual salivary $\alpha$ amylase that was collected from the sublingual gland, look at Figure 3.

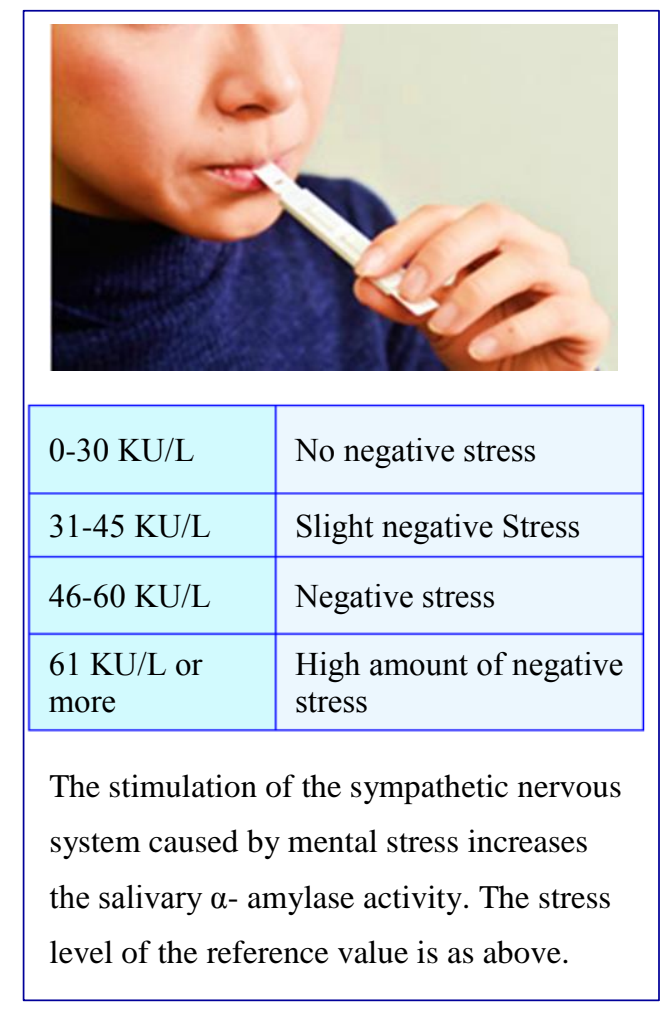

Figure 3: The active amount of $\alpha$ amylase in the saliva. Authority: NIPRO.

\subsection{The method of analyzation}

We compared the score of the MoCA test and the amount of sublingual salivary amylase that is before intervention, during the 3 months of non-intervention, and after the intervention through the use of one-way analysis of variance (ANOVA). After that, we clarified whether there is a relevance between cognition score and the stress level by using the Pearson's correlation coefficient.

\subsection{Ethical consideration}

We received the admission from the organizations that the researchers belong to and the ethical review board. We accepted the participation by the submission the letter of consent and the ones who understood the explanation of the purpose and the method, the free to participate and to exercise their veto, the protection of privacy, the way we manage the data, and we announced the result orally and through documents.

\section{Result}

For analyzed the result of 108 participations out of 162 people who have registered. The average age of the subjects were $75 \pm 8.2$ years old and 19 people were male, and 89 people were female. There are significant differences 
among the following respects, which are the result of one-way analysis of variance, the average score of the MoCA test, which is the evaluation of the cognitive function, the score that shows the score of repeated tasks of immediate reproduction ability, the score of delay recall, the total score (cut off score: 26 points) that distinguish whether there is a doubt on mild cognitive impairment, and the amount of sublingual salivary amylase, which shows the stress level.

The average score of the repeated tasks were 0.82 before the intervention, 0.62 during the intervention, and 0.97 (out of 1) after the intervention. It showed $\mathrm{F}(2,141)=3.85, \mathrm{p}=0.039$ among the variance analysis and the multiple comparison, which used the Tukey, and there was an increase in the scores between after the non-intervention term and after the intervention ( $\mathrm{p}=0.049$, Figure 4 ). The average score of delayed recall was 3.38 before the intervention, 3.19 during the intervention, 4.36 (out of 5) after the intervention. It showed $\mathrm{F}(2,141)=14.79, \mathrm{p}=0.000$ among the variance analysis and the multiple comparison, which used the Tukey, and there was an increase in the scores respectively between before and after intervention $(\mathrm{p}=0.000)$, and between after non-intervention term and after intervention ( $\mathrm{p}=0.000$, Figure 5).

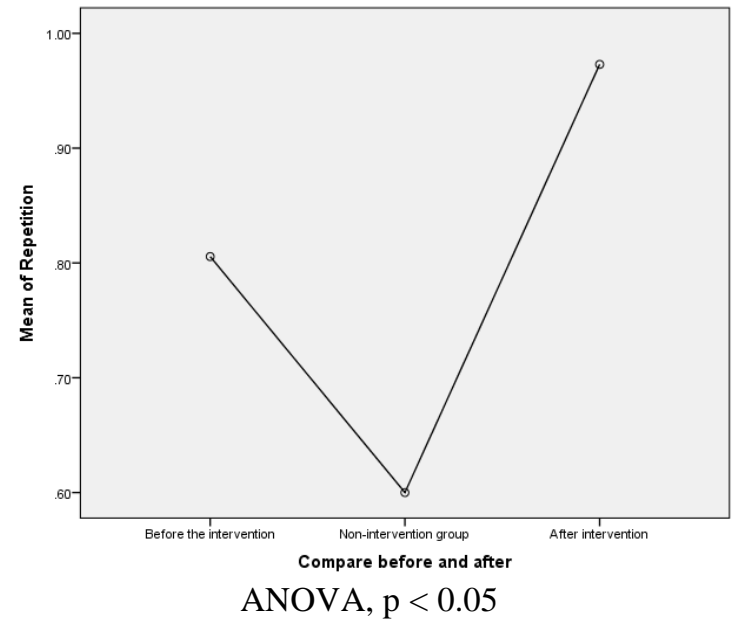

Figure 4: The comparison between the average score of repeated tasks before and after intervention.

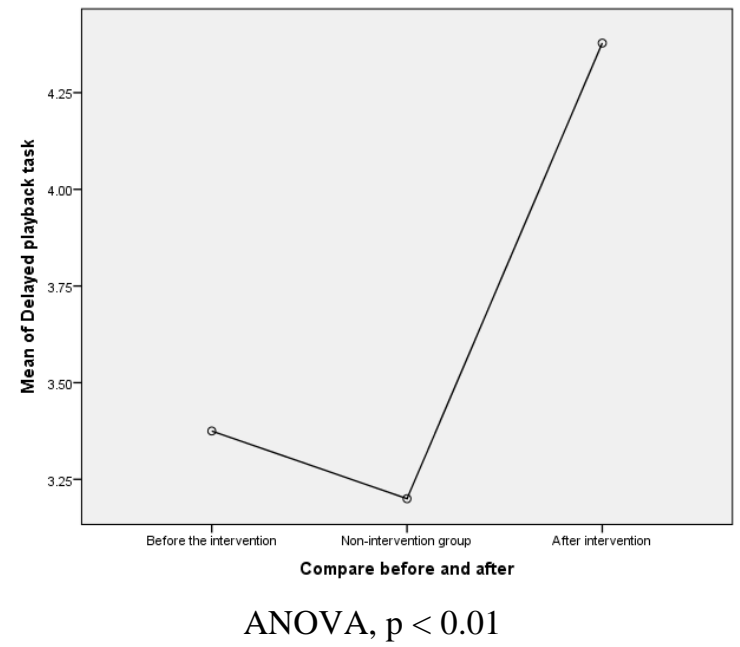

Figure 5: The comparison between the average score of delay recall tasks before and after the intervention. 
The average total score that distinguishes whether they have the possibility of mild cognitive impairment or not is 23.9 before the intervention, 23.4 during the non-intervention term, and 25.8 (out of 30) after the intervention. It showed $\mathrm{F}(2,141)=9.67, \mathrm{p}=0.002$ among the variance analysis and the multiple comparison, which used the Tukey, and there was an increase in the scores respectively between before and after the intervention ( $\mathrm{p}=0.003)$, and between after the non-intervention term and after the intervention ( $p=0.000$, Figure 6). Even though the score before the intervention and after the non-intervention term scored more than 2 points lower than the cut off value, after the intervention it scored to be 0.2 until the cut off amount.

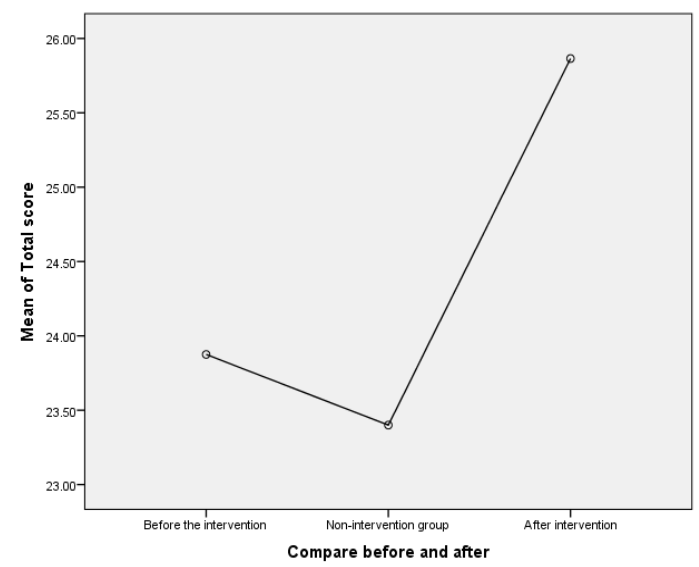

ANOVA, $\mathrm{p}<0.01$

Figure 6: The comparison between the average score of the total score before and after the intervention.

The average amount of sublingual salivary $\alpha$ amylase was 53.9 before the intervention, 49.6 during the nonintervention term, and 40.5 after the intervention. It showed $\mathrm{F}(2,141)=4.89, \mathrm{p}=0.028$, among the variance analysis and the multiple comparison, which used the Tukey and there a significant decrease in the stress level between before and after the intervention ( $\mathrm{p}=0.047$, Figure 7).

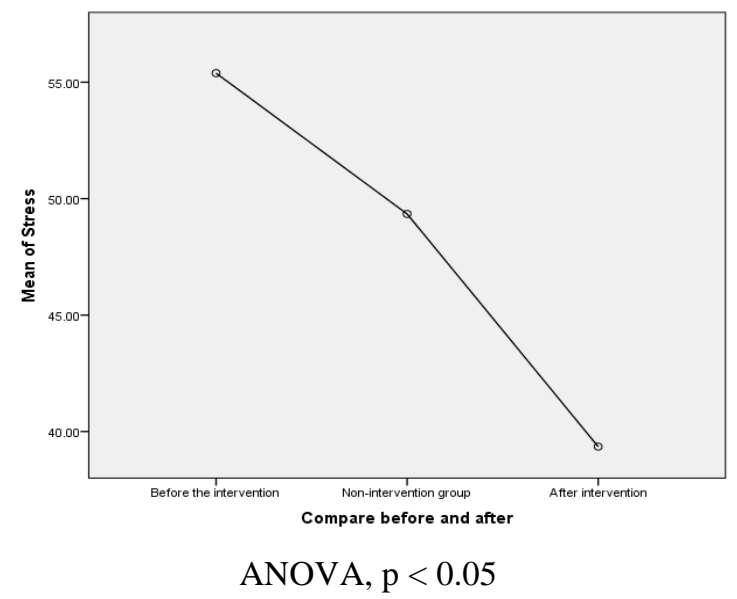

Figure 7: The comparison between the amount of $\alpha$ amylase in the saliva (stress level) before and after the intervention. 
The value of $\alpha$ amylase before and after the non-intervention term, resulted in a consequence, which said "having stress", but the value scored 40.5 after the intervention and improved to a result that says "having a little stress".

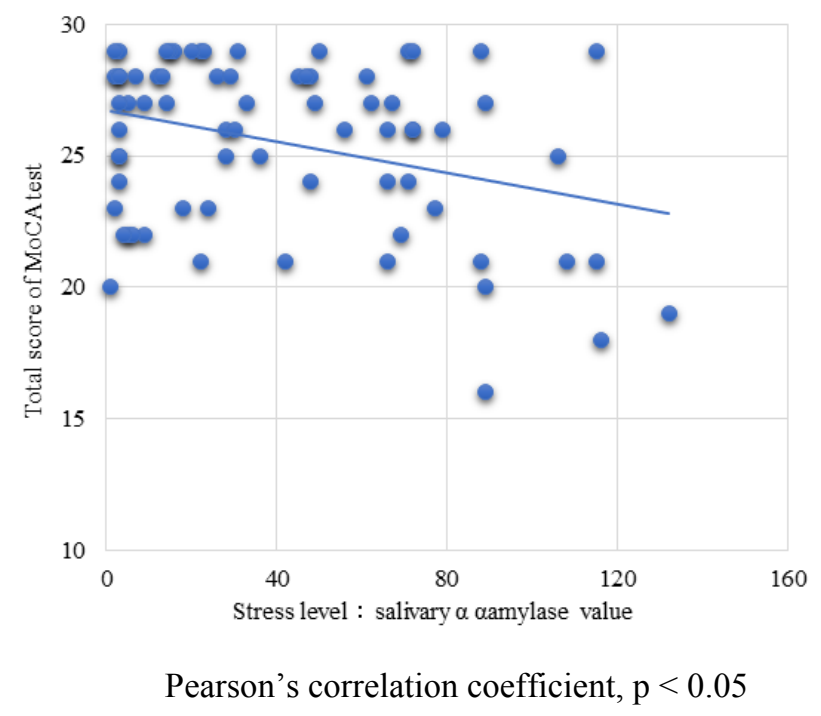

Figure 8: The correlation between the cognitive score and the stress level.

As it can be seen in Figure 8, there is a negative correlation between the correlation between the stress level (level of $\alpha$ amylase) and the score of cognitive function and the more the stress level was low, did the cognitive scored higher. (Pearson's correlation coefficient; $r=-0.37$ ).

\section{Discussion}

The method that developed for this research is to repeat the short term of musical phrases and the choreography several times and make them reconstruct their memories after the music therapy. Moreover, we change the choreography during the music and after that we conducted delayed recall. This has the characteristic that by memorizing along with the music, we can easily memorize and recall the phrases or the movements. Lyrics indicated by songs can be remembered much better than clauses indicated by speech [34]. In addition, among the memory recall tests the group that done musical training scored better than the group that did not do the training. Moreover, the MRI showed that the domain of the brain was wider of the group that did the musical training and the possibility in the improvement of the cognitive function is suggested [35]. Furthermore, active music therapy: AMT is more efficient to the memory than just listening to music passively and the improvement in the working memory is verified [36-38]. Working memory is a memory function that Baddeley proposed in 1986, and it is an ability to handle the temporary memory that is necessary when working or acting [39]. It is also used in our daily conversation, reading and writing, and calculation. Our daily life is composed by the repeat of the working memory and it is thought that if this function is maintained it is easier to maintain our daily function too. Based on these leading research, we combined the music therapy and memory tasks and intervened and compared them with the group of non-intervention. We compared the result of the evaluation of cognitive function by the use of MoCA test, which is a screening test of mild cognitive impairment. 
As a result, among the total score, the cognitive score of immediate replay tasks and delay recall tasks, increased significantly after the intervention and especially the increase in the score of the ability of delay recall was the most extended. The total score of whether there is a possibility of MCI, the score before the intervention and the group non-intervention scored more than 2 points lower than the cut off value, but it became approached to a score that is 0.2 points until the cut off value after the intervention. From this result, it was revealed that the efficiency of the ability of delay recall, which is the purpose and it is conceived that it can help the maintain and improve the working memory, which is used to work with keeping the memory from shortly before. The second purpose, which is about the stress level, the amount of aamylase were 53.9 and 49.6 before the intervention and the group of nonintervention showed that it is stressed, but after the intervention, it decreased to 40.5 and showed that it stressed a little. In addition, there was a negative correlation between the stress level and the score of cognitive functions and it showed that as the stress level is low, the cognitive score was high. It was known that under the situation with stress it leads to low concentration, and as the stress level is low, the grade of memory tasks became high, but also in this research the correlation was also verified.

From this result it is thought that it is necessary to reduce stress in order to improve memory ability and it is suggested that relaxation effect [41-42], which is caused by the music therapy shows a positive impact on our memory. Many of the intervention that uses the help of music, has the purpose of exercising by moving along with the rhythm, like TV gymnastics, but it is also said that not only do they have physical effect, but also mental effects [43]. Moreover, there is an increase in the ways of intervention such as exercising and singing along, which are called "gymnastics in the old song" that aims the preference of elderly people. In addition to physical effects, these ways has the purpose to gain mental effects such as the recall of the good old memories by the songs that are familiar with the elderly people, the stabilization of the emotions by the work of music [44]. It can be seen in our intervention method that by conducting a choreography that goes along with the music it has physical effects and mental effects by music, and there is an effect in cognitive functions by the delay recall tasks, and it also showed the improvement in the cognitive functions and the reduction in stress. We conceive that the theme from now on is to verify through interviewing the effect in daily life and to improve the precision so that there will be a positive effect for the daily lives of elderly people.

\section{Conclusion}

We verified the effect of the method of cognitive training, which the delay recall tasks was combined with the music therapy, and found that there was a significant improvement in the cognitive function. In addition, by using this method, there is a benefit that the memory training can be conducted non-stressfully for the elderly people. Future task is to continue and expand this method.

\section{Acknowledgement}

We appreciate to everyone from the bottom of our heart to the elderly people, the local self-government association and everyone who is concerned with the council of social welfare, and the therapists of music therapy. 


\section{Conflict of Interests}

In this research, we conducted this research by the cooperation of the group of music therapy, but we do not have the conflict of interests.

\section{References}

1. Burns A, Iliffe S. Alzheimer's disease. BMJ 338 (2009): 158.

2. Cabinet Office. Current situation and trend of the environment surrounding the elderly. Aging Society White Paper (2016): 19-23.

3. Holmes C, Boche D, Wilkinson D, et al. Long-term effects of Abeta42 immunisation in Alzheimer's disease: follow-up of a randomised, placebo-controlled phase I trial. Lancet 372 (2008): 216-223.

4. World Health Organization: Fact sheets/Dementia. http://www.who.int/en/news-room/factsheets/detail/dementia. 2018/08/02

5. Al-Yahya E, Johansen-Berg H, Kischka U, et al. Prefrontal Cortex Activation While Walking Dual-Task Conditions in Stroke: A Multimodal Imaging Study. Neurorehabilitation and Neural Repair 30 (2015): 591599.

6. Ohsugi $\mathrm{H}$, Ohgi S, Shigemori $\mathrm{K}$, et al. Differences in dual-task performance and prefrontal cortex activation between younger and older adults. BMC Neuroscience 14 (2013): 1-9.

7. Owen AM, McMillan KM, Laird AR, et al. N-back working memory paradigm: A meta-analysis of normative functional neuroimaging studies. Hum Brain Mapp 25 (2005): 46-59.

8. Yogev-Seligmann G, Hausdorff JM, Giladi N, et al. The role of executive function and attention in gait. Mov Disord 23 (2008): 329-342.

9. Holtzer R, Mahoney JR, Izzetoglu M, et al. fNIRS study of walking and walking while talking in young and old individuals. J Gerontol A Biol Sci Med Sci 66 (2011): 879-887.

10. Anguera JA, Boccanfuso J, Rintoul JL, et al. Video game training enhances cognitive control in older adults. Nature 501 (2013): 97-101.

11. Kirchner WK. Age differences in short-term retention of rapidly changing information. J Exp Psychol 55 (1958): 352-358.

12. Susanne JM, Studer-Luethi B, Buschkuehl M, et al. The relationship between n-back performance and matrix reasoning-implications for training and transfer. Intelligence 38 (2010): 625-635.

13. McNab F, Varrone A, Farde L, et al. Changes in Cortical Dopamine D1 Receptor Binding Associated with Cognitive Training. Science 323 (2009): 800-802.

14. Owen AM, McMillan KM, Laird AR, et al. N-back working memory paradigm: a meta-analysis of normative functional neuroimaging studies. Hum Brain Mapp 25 (2005): 46-59.

15. Snyder R. Music and Memory-An Introduction. A Bradford Book (2001): 47-74.

16. Raglio A. Dementia in Music Therapy. Non-Pharmacological Therapies in Dementia 1 (2010): 1-14.

17. Gomez Gallego M, Gomez Garcia J. Music therapy and Alzheimer's disease: Cognitive, psychological, and behavioural effects. Neurologia 32 (2017): 300-308. 
18. Fang R, Ye S, Huangfu J, et al. Music therapy is a potential intervention for cognition of Alzheimer's Disease: A mini-review. Transl Neurodegener 6 (2017): 1-8.

19. Ozdemir L, Akdemir N. Effects of multisensory stimulation on cognition, depression and anxiety levels of mildly-affected Alzheimer's patients. J Neurol Sci 283 (2009): 211-213.

20. Sarkamo T, Tervaniemi M, Laitinen S, et al. Cognitive, emotional, and social benefits of regular musical activities in early dementia: randomized controlled study. Gerontologist 54 (2014): 634-650.

21. Herholz SC, Herholz RS, Herholz K. Non-pharmacological interventions and neuroplasticity in early stage Alzheimer's disease. Expert Rev Neurother 13 (2013): 1235-1245.

22. Simmons-Stern NR, Budson AE, Ally BA. Music as a memory enhancer in patients with Alzheimer's disease. Neuropsychologia 48 (2010): 3164-3167.

23. King JB, Jones KG, Goldberg E, et al. Increased Functional Connectivity After Listening to Favored Music in Adults With Alzheimer Dementia. The Journal of Prevention of Alzheimer's Disease (2018).

24. Music and Memory: About MUSIC and MEMORY® Certification Program https://musicandmemory.org/training-publications/whats-included/ (2018).

25. Meeusen R, Piacentini MF, Kempenaers F, et al. Brain neurotransmitter levels during exercise. Dtsch Z Sportmed 52 (2001): 361-368.

26. Ohmatsu S, Nakano H, Tominaga T, et al. Activation of the serotonergic system by pedaling exercise changes anterior cingulate cortex activity and improves negative emotion. Behav Brain Res 270 (2014): $112-117$.

27. Meneses A, Liy-Salmeron G. Serotonin and emotion, learning and memory. Rev Neurosci 23 (2012): 543553.

28. Meneses A. Serotonin, neural markers, and memory. Front Pharmacol 6 (2015): 1-22.

29. The Carnegie Hall Corporation. The Orchestra Rocks with Ostinato Focal Work. Steve Reich's Clapping Music (2018): 16-31.

30. Lange MD. Teaching the ostinati patterns. Together in Harmony. Combining Orff Schulwerk and Music Learning Theory (2005): 54-58.

31. McEwen BS. Central effects of stress hormones in health and disease. Understanding the protective and damaging effects of stress and stress mediators. Eur J Pharmacol 583 (2008): 174-185.

32. Khalfa S, Bella SD, Roy M, et al. Effects of relaxing music on salivary cortisol level after psychological stress. Ann NY Acad Sci 999 (2003): 374-376.

33. Labbe E, Schmidt N, Babin J, et al. Coping with Stress: The Effectiveness of Different Types of Music. Applied Psychophysiology and Biofeedback 32 (2007): 163-168.

34. Wallace WT. Memory for Music: Effects of Melody on Recall of Text. Journal of Experimental Psychology. Learning, Memory, and Cognition 20 (1994): 1471-1485.

35. Chan A, Ho Y, Cheung M, et al. Music training improves verbal memory. Nature 396 (1998): 128.

36. Baddeley A. Working Memory: Theories, Models, and Controversies. Annu Rev Psychol 63 (2012): 1-29.

37. Rao TI, Nagendra HR. The effect of active and silent music interventions on patients with type 2 diabetes measured with electron photonic imaging technique. Int J Humanit Soc Sci 3 (2014): 7-14. 
38. George E, Coch D. Music training and working memory: an ERP study. Neuropsychologia 49 (2011): 1083-1094.

39. Suarez L, Elangovan S, Agnes Au. Cross-sectional study on the relationship between music training and working memory in adults. Aus J Psychol (2015): 1-9.

40. Smeets T, Jelicic M, Merckelbach H. The effect of acute stress on memory depends on word valence. International Journal of Psychophysiology 62 (2006): 30-37.

41. Khalfa S, Bella SD, Roy M, et al. Effects of relaxing music on salivary cortisol level after psychological stress. Ann NY Acad Sci 999 (2003): 374-376.

42. Blood AJ, Zatorre RJ. Intensely pleasurable responses to music correlate with activity in brain regions implicated in reward and emotion. Proc Natl Acad Sci USA 98 (2001): 11818-11823.

43. Watabe R, Aoyama T. Research on the effect of radio gymnastics on physical function. Health Support Study Group of Kanagawa University of Health and Welfare (2009): 1-37.

44. Ito A, Isowa T. Effects of music intervention to encourage recollection for elderly people with dementia About changes in physical and mental functions and social characteristics-Proceedings of the Japanese Nursing Association 42 (2012): 272-275.

Citation: Kazue Sawami, Tetsuro Kitamura, Chizuko Suishu. Effect of Cognitive Training by Music Therapy. Journal of Psychiatry and Psychiatric Disorders 2 (2018): 167-178.

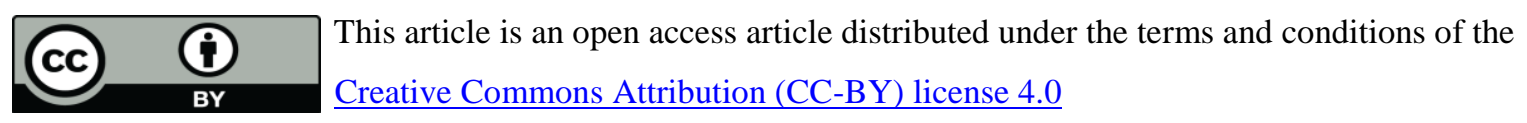

